\title{
The CMS Central Hadron Calorimeter
}

\author{
Jim Freeman \\ For the E892 Collaboration \\ Fermi National Accelerator Laboratory \\ P.O. Box 500, Batavia, Illinois 60510
}

December 1996

Proceedings of the IV International Conference on Calorimetry in High Energy Physics, Frascati, Italy, June 8-14, 1996 


\section{Disclaimer}

This report was prepared as an account of work sponsored by an agency of the United States Government. Neither the United States Government nor any agency thereof, nor any of their employees, makes any warranty, expressed or implied, or assumes any legal liability or responsibility for the accuracy, completeness, or usefulness of any information, apparatus, product, or process disclosed, or represents that its use would not infringe privately owned rights. Reference herein to any specific commercial product, process, or service by trade name, trademark, manufacturer, or otherwise, does not necessarily constitute or imply its endorsement, recommendation, or favoring by the United States Government or any agency thereof. The views and opinions of authors expressed herein do not necessarily state or reflect those of the United States Government or any agency thereof.

\section{Distribution}

Approved for public release; further dissemination unlimited. 


\title{
The CMS Central Hadron Calorimeter
}

\author{
Jim Freeman \\ Fermi National Accelerator Laboratory, Batavia, Illinois 60510 \\ Representing the CMS Hadron Calorimeter Group
}

\begin{abstract}
The CMS central hadron calorimeter is a copper absorber/ scintillator sampling structure. We describe design choices that led us to this concept, details of the mechanical and optical structure, and test beam results. We discuss calibration techniques, and finally the anticipated construction schedule.
\end{abstract}

\section{Overview}

The CMS detector is a general purpose experiment that will operate at the Large Hadron Collider at CERN. It places an emphasis on electron and photon energy resolution and muon identification. A key to achieving these goals is to have very large very strong magnetic field volume for momentum measurement, and to have a high precision electromagnetic calorimeter. The heart of the CMS detector is a large volume $4 \mathrm{~T}$ superconducting solenoid. The solenoid is 13 meters long with an inner radius of 3 meters. Inside the solenoid are placed tracking detectors, followed by an electromagnetic calorimeter (ECAL) made of lead tungstate crystals and finally the hadron calorimeter (HCAL). Among the reasons for placing the HCAL inside the solenoid was the desire to continue muon bending through the hadron calorimeter to improve muon momentum measurement and triggering. Placing the HCAL behind a greater than $1 \lambda$ thick solenoid would have also seriously degraded the performance. Figure 1 shows a quarter section of the CMS detector design.

A major function of the HCAL is missing transverse energy measurement. For this measurement, gausssian resolution is not as important as elimniation of low energy tails in the response function. With this in mind, the CMS HCAL design strives to eliminate dead material that causes energy loss, and to maximize the calorimeter $\lambda$.

The location of the HCAL inside the magnetic field required that the calorimeter be non-magnetic. In addition, the constrained space inside the solenoid pointed toward a design that had a compact interaction length. Two reasonable choices for absorber were stainless steel and copper. Copper, with its approximately $10 \%$ shorter interaction length was chosen for the absorber. 


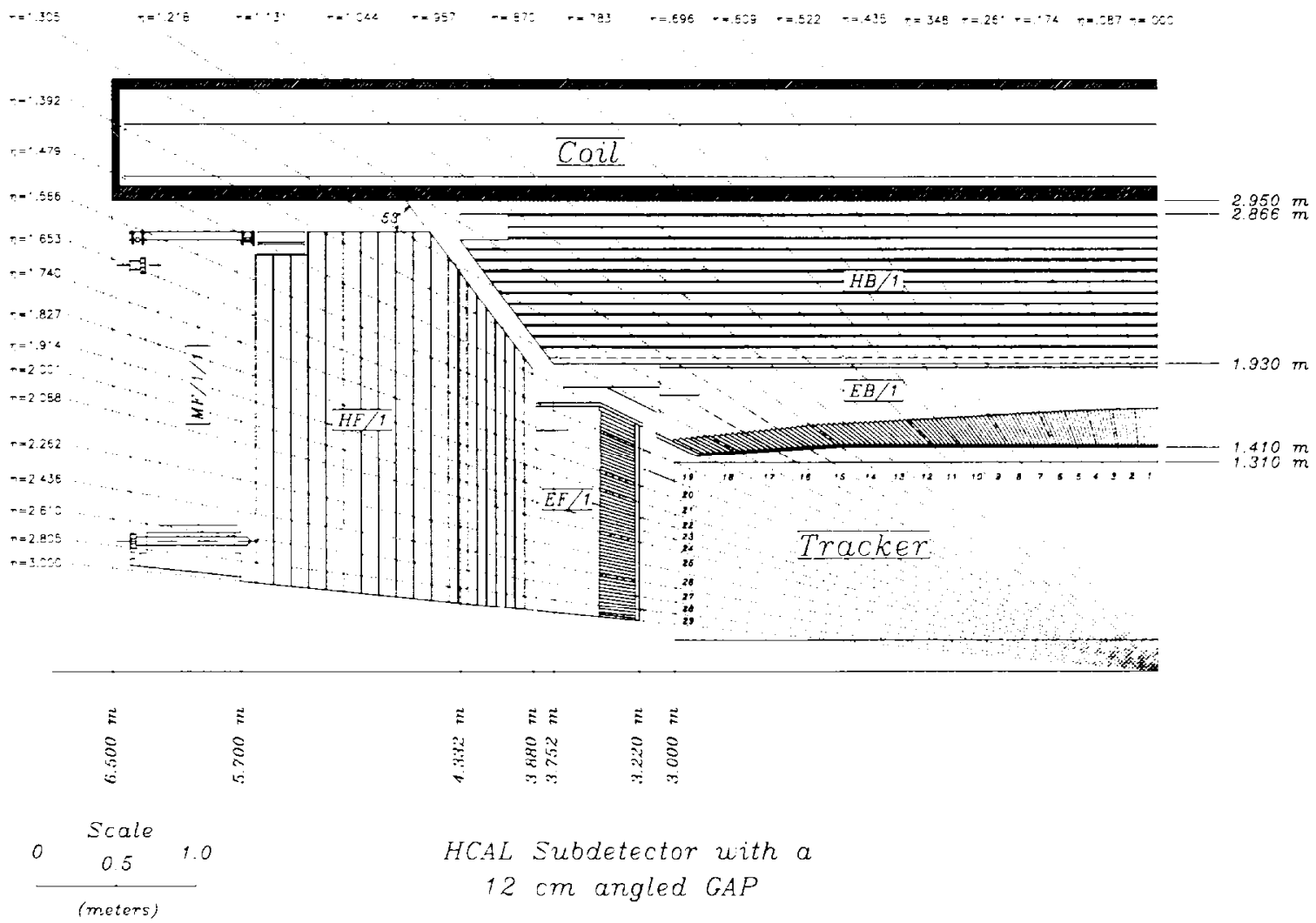

Figure 1: Quarter-section of the CMS central detector.

The central HCAL covers the $\eta$ range of $-3<\eta<3$ and $0<\phi<2 \pi$. The central HCAL is physically composed of 2 regions, the barrel $(|\eta|<1.4)$ and the endcap, which extends to $|\eta|=3.0$. The very forward region of CMS , $3<$ $|\eta|<5$, is covered by a quartz fiber calorimeter, discussed by V. Gavrilov in these proceedings 1 ). The tower granularity is chosen to be $\Delta \eta \times \Delta \phi=0.087 \times 0.087$. The segmentation is commensurate with the granularity of ECAL, and is also sufficient for jet reconstruction. 2)

The tile/fiber sampling technique was chosen for calorimeter design. This technique uses scintillator tiles to sample the shower. Wave-shifting fiber imbedded in the tile traps the scintillator light and clear fiber spliced to the WLS fiber carries the light to photo-transducers. First developed at Protvino and CERN 3), the technique has been enhanced and applied to the CDF endcap upgrade calorimeter at Fermilab 4). In this design, the samples are thin. They require only $0.9 \mathrm{~cm}$ 
of thickness between absorber plates, so a high density calorimeter is maintained. The choice of scintillator for readout provides a calorimeter that is fast, stable and reliable, and radiation-resistant. More than $90 \%$ of the scintillation light will be collected within a $50 \mathrm{~ns}$ time window. Scintillators have been developed that survive the radiation exposure that 10 years of operation at the LHC will create ( 2 - 4 Mrads, at the worst location, the $|\eta|=3$ corner of the endcap hadron calorimeter).

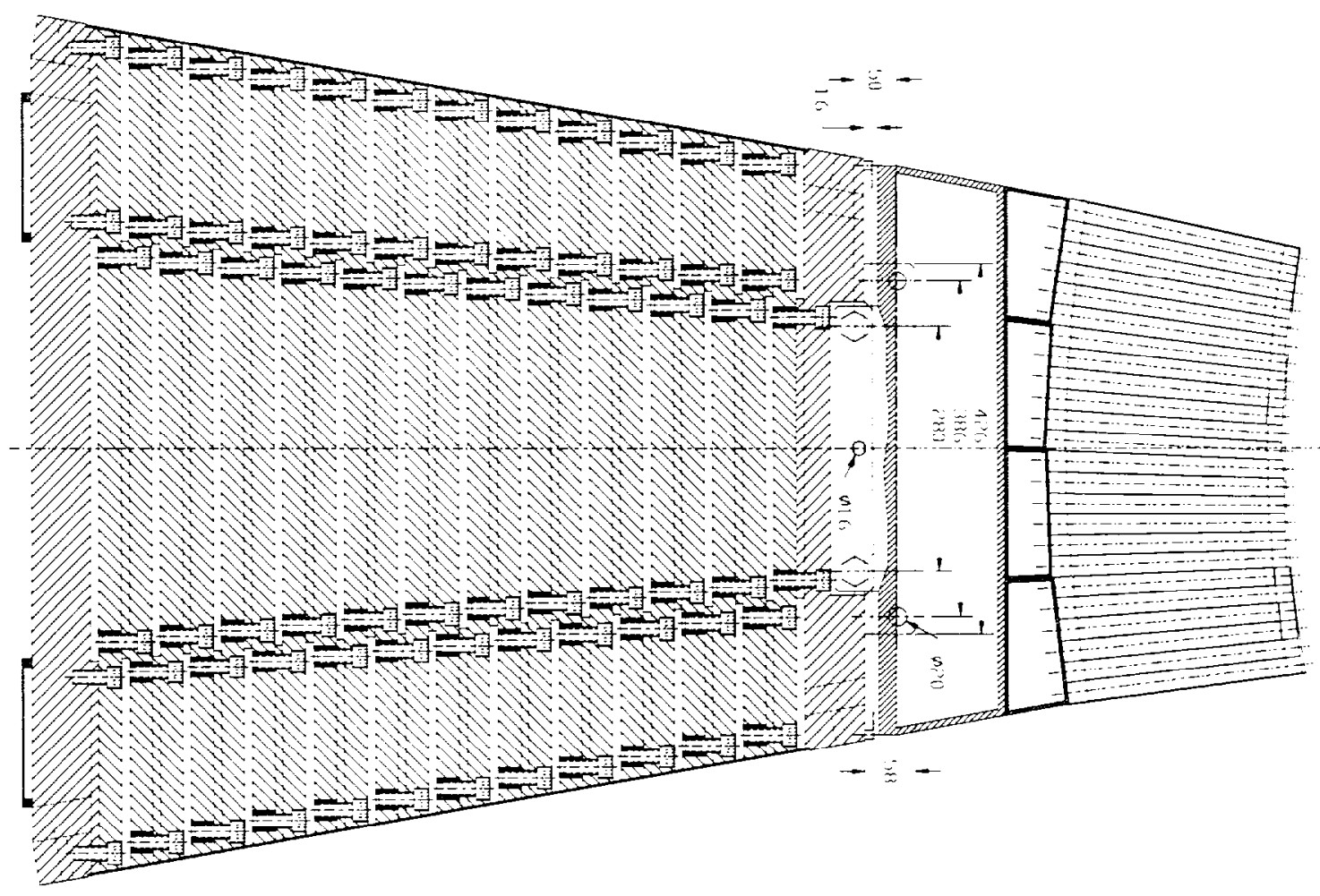

Figure 2: Barrel wedge mechanical construction.

\section{Mechanical Design}

Figure 2 shows the mechanical structure of a module of the central HCAL barrel. The barrel HCAL is made of $\delta(\phi)=20^{\circ}$ wedges. Each wedge extends from $\eta=0$ to the high $\eta$ boundary, $\eta \simeq 1.4$. A wedge is about 4.5 meters long, and weighs about 27 metric tons. A wedge is composed of $6 \mathrm{~cm}$ thick inner and outer stainless steel plates (for mechanical strength), with $6 \mathrm{~cm}$ thick copper absorber samples inside. The entire structure is bolted together. Figure 2 also shows the $\delta(\phi)=20^{\circ} \mathrm{ECAL}$ module attached to the front of the HCAL wedge.

The absorber structure is designed with alternating "staggered" slots for 
the scintillators. Thus the ( 4 tower wide in $\phi$ ) wedge starts with a slot to accommodate scintillators the middle 2 towers, then $1 / 2$ of an absorber sample later in depth, have separate slots for the outer 2 towers. The staggering of the absorber plates provides a rigid mechanical structure, with no projective dead regions. Each slot runs the full length (in $\eta$ ) of the wedge. Long thin scintillator "tile trays" will be inserted from the high $\eta$ end, and optical cables will carry the scintillation light to the photodetectors. 18 wedges are bolted together to form a half-barrel ring. Tight mechanical tolerances on the plates (and lack of distortion because of bolting) allows the design to have $\leq 2 \mathrm{~mm}$ of air gap between adjacent wedges. In addition, only a $9 \mathrm{~mm}$ high slot is needed to accommodate the $7.5 \mathrm{~mm}$ thick scintillator packages.

The construction endcap hadron calorimeter is logically similar to the barrel. Each endcap is a monolithic structure, bolted together from "pizza slice" shaped plates of copper. Again the plates are staggered to provide alternating slots for the scintillator tile trays. The endcap HCAL is anticipated to have $\sim 10 \mathrm{~cm}$ thick copper sampling.

Figure 3 shows the total number of interaction lengths of material provided by the ECAL $(1.1 \lambda)$ and the HCAL. We see that at $\eta=0$, the combined calorimeter is somewhat thin, $6.2 \lambda$. Therefore our design places 2 additional scintillator samples outside the solenoid, the Late Shower Sample. Figure 3 also shows the resulting total number of interaction lengths in the calorimeter.

\section{Optical System}

The CMS HCAL optical system contains of approximately 70,000 individual scintillator tiles. Since the optical systems for the barrel and endcap HCAL's are very similar, we will concentrate on the description of the barrel.

The scintillators are organized into tile trays, as shown in Figure 4 for the barrel calorimeter. The trays are either 1 or 2 tiles wide in $\phi$, and the full length of the barrel (16 tiles) in $\eta$. The cross section of the tile tray is shown in Figure 5 . The $4 \mathrm{~mm}$ thick scintillator tiles are covered with white reflective Tyvek plastic and then sandwiched between top and bottom cover plates. The entire package is connected together by small through-bolts. The thin bottom cover plate provides mechanical protection. The $2 \mathrm{~mm}$ thick top plate protects the tiles as well as supplies a path for the fibers from the tiles to travel to the high $\eta$ end of the tile tray. There, the fibers are terminated into multi-fiber optical connectors. Optical cables carry the light onward to photodetector decoder boxes where the light from each tile is organized into readout towers.

Our baseline choices for optical materials are Kuraray SCSN81 for the scintillator, Kuraray multi-clad Y-11 (K-27 fluor) for the wave-shifting fiber, and 


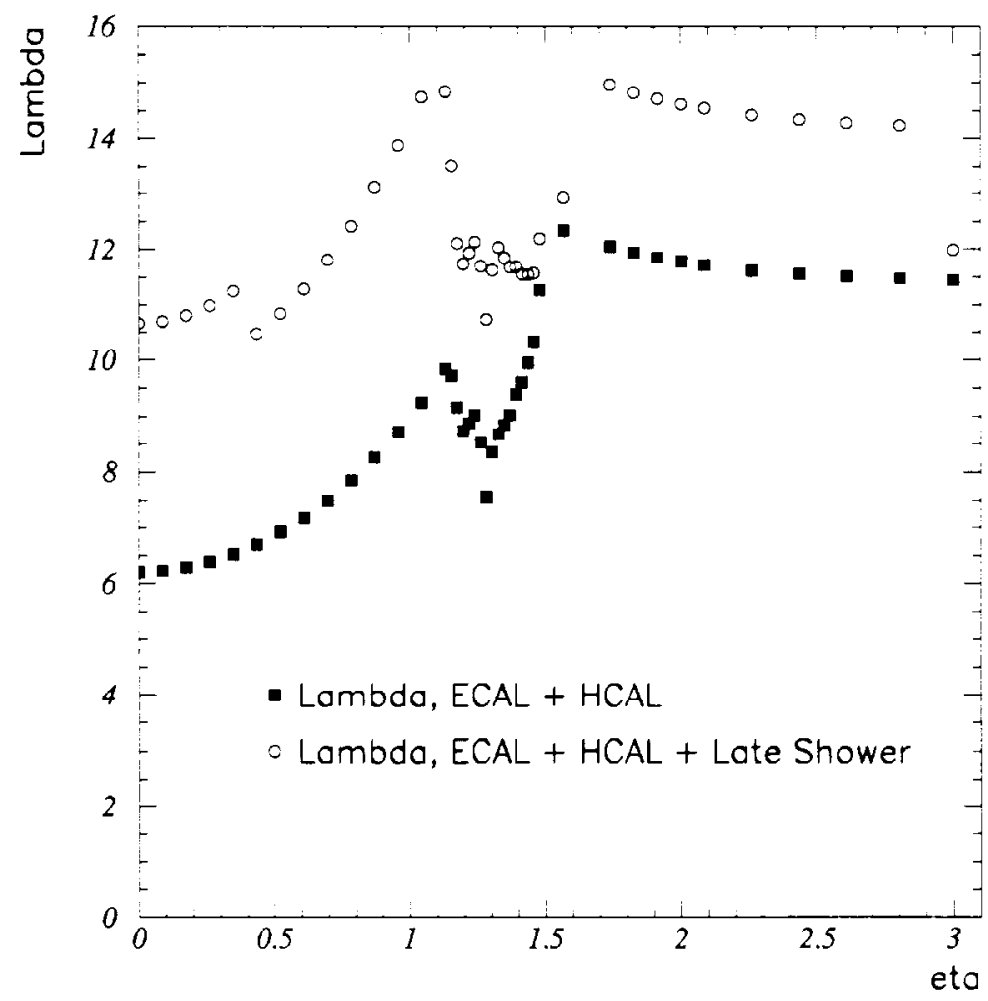

Figure 3: Number of interaction lengths inside the solenoid (ECAL + HCAL), and total number of interaction lengths sampled, including the Late Shower Sample.

Kuraray multi-clad clear fiber. With these materials, typical light-yields are of order 2 photoelectrons per minimum-ionizing particle per scintillator layer. Optical materials evaluation will continue for the next several months before the final choices are made.

\section{Quality Control and Calibration}

Quality control and calibration schemes are built into the optical system from the beginning. After assembly of the tile trays a collimated $C \boldsymbol{s}^{137}$ source is used to test the tiles. By measuring the induced radioactive source current after a photodetector, the collimated source measurement establishes the absolute response of the tiles. At the same time a moving wire source is used for cross calibration. Figure 5 shows the $2 \mathrm{~mm}$ top cover plate carrying "source tubes". The source tubes are stainless steel tubes that terminate into source-tube connectors that allow them to be to "plumbed" to tubes from a moving wire source system. In the moving wire source system, a point source at the end of a wire moves through the source tubes to excite the scintillators. The source position relative to the tile is well controlled by the 


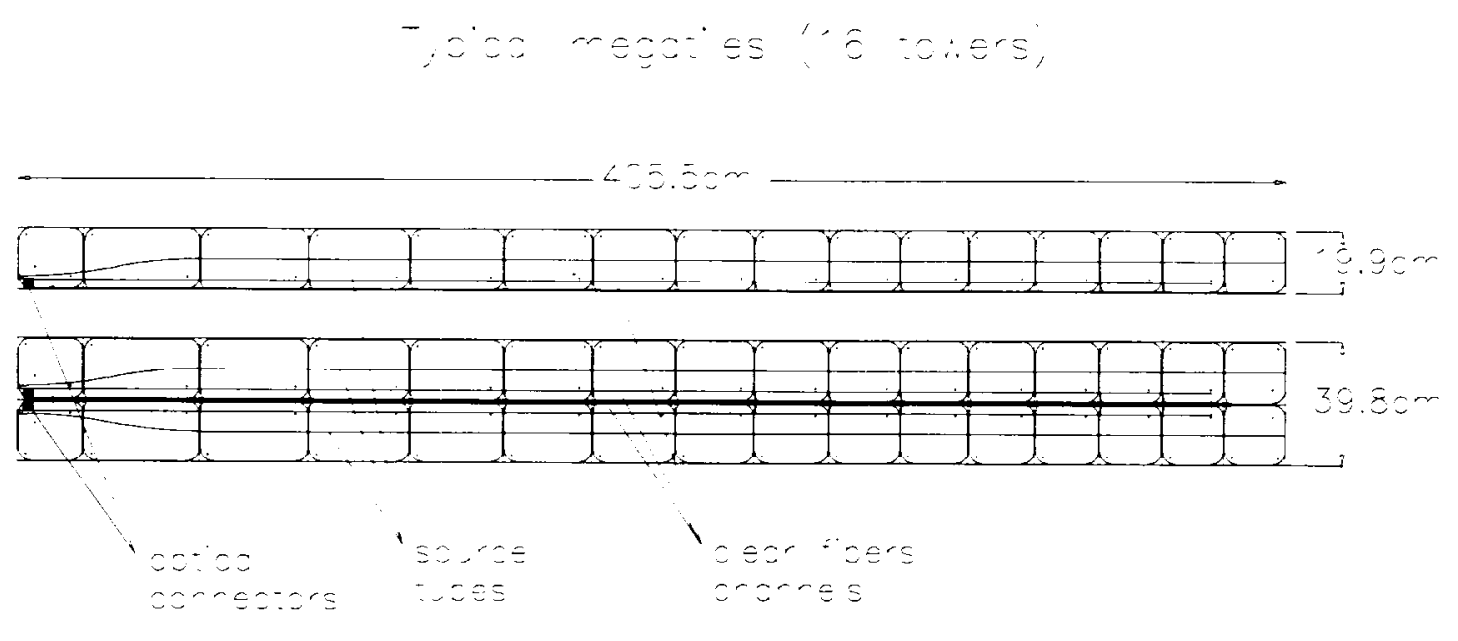

Figure 4: Schematic drawing of a scintillator tile tray. The tray is 16 tiles long in $\eta$ and either 1 or 2 tiles wide in $\phi$.

permanently attached source tube. Because of the fixed geometry of the source tube relative to the tile, the ratio of response of collimated to wire source is stable. The moving wire source $\left(C s^{137}\right)$ is basically an isotropic source. The scintillator response changes by approximately $1 \%$ per $0.1 \mathrm{~mm}$ of separation between the scintillator and the source. Thus meaningful measurements with the wire source require that the stability of the placement of the wire source relative to scintillator must be controlled to order $0.1 \mathrm{~mm}$. This is achieved by permanent attachment of the source tubes on the tile trays.

After the tile-trays have been installed into the absorber, the wire source is again used to test for system stability. Since the geometry of the wire source tube to scintillator is unchanged, the measurement of wire-source response allows for reference back to the original QC test using the collimated source. A small number of the source tubes will be accessible during operation of CMS. These tubes will be periodically retested to verify stability.

A laser flasher system will also be used to directly excite the photodetectors. This system will be used on a periodic basis to track the gain of the photodetector/amplifier/digital readout system.

The stability of ratio of wire source measurement to collimated source measurement (ie to the true tile absolute response) provides convenient way to carry test beam calibrations to the actual CMS detector. A subset of the barrel wedges will be extensively studied in test beam. A set of calorimeter towers will have their pion response measured with test beams. In addition the wire-source system will measure 


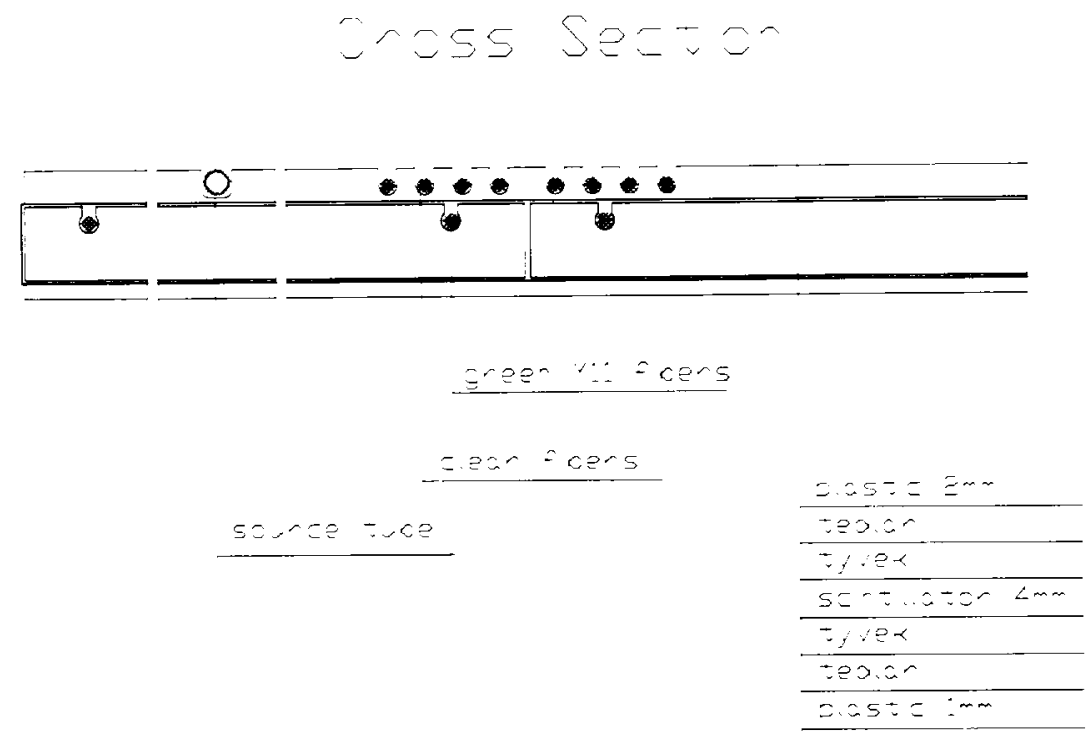

Figure 5: The cross section of the scintillator tile tray.

the "fingerprint" of each tower's optical longitudinal uniformity. An effective "pionweighted source response" will be formed by convoluting the longitudinal optical profile with an average pion longitudinal profile. Then the ratio of actual test beam response to pion-weighted source response will be used to carry test beam calibration to wedges in CMS that were not exposed to beams. From past experience on CDF, an initial absolute calibration of $2-3 \%$ is expected.

The HCAL group has started a program to understand how the intial absolute calibration can be improved by using in-situ physics calibrations while CMS is operating 5 ). One likely signal is $t \bar{t}$ production, where the top quarks then decay into $\mathrm{W}+\mathrm{b}$. One $\mathrm{W}$ is required to decay into jets, while the other is required to decay into lepton + neutrino to provide a trigger. For events that have 2 tagged b-jet's, CDF has shown that it can readily reconstruct the $\mathrm{W}$ boson that decays into jets. They measure a rms/mean of about $9.1 \mathrm{GeV} / 81.4 \mathrm{GeV}$ for 8 reconstructed top events in $100 \mathrm{pb}^{-1}$ of data. A similar analysis has been performed using a simulation of the CMS detector. There, even in the presence of the $\sim 30$ minimum-bias events anticipated at the ultimate luminosity of $10^{34}$, the $\mathrm{W}$ into 2 -jet decay can be reliably reconstructed. The results expected for one month of LHC running at $10^{33}$ is shown in Figure 6. At the LHC, $t \bar{t}$ production will supply a ready source of di-jet events that reconstruct into a fixed mass and will help with calibration as well as understanding systematics of jet clustering. 


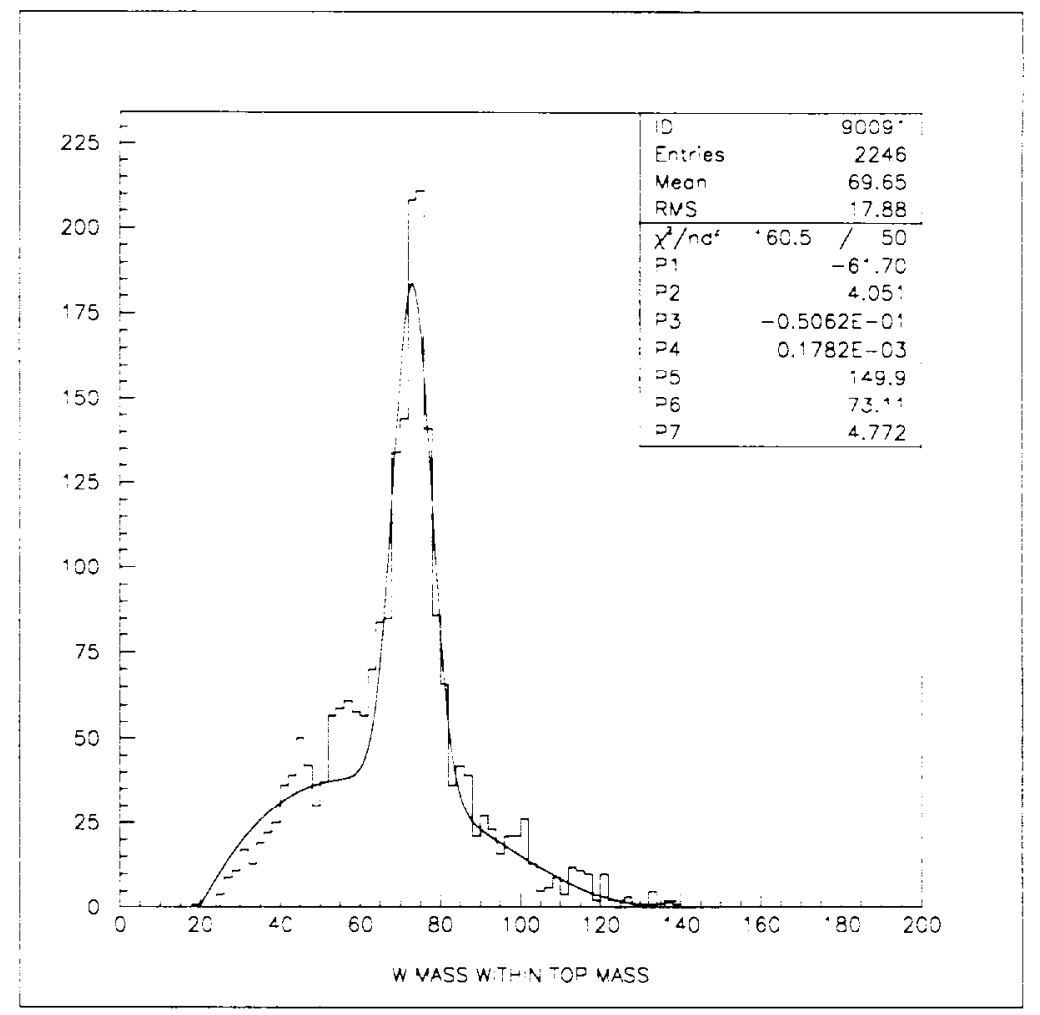

Figure 6: Simulated reconstruction of $\mathrm{W}$ decaying into 2 jets for double-b-tagged $t \bar{t}$ events in CMS. The statistics represent one month of low-luminosity running at the LHC.

\section{Photodetectors}

The barrel photodetectors are positioned at the high $\eta$ / large radius of the barrel HCAL, inside the $4 \mathrm{~T}$ magnetic field. (The corresponding location for photodetectors for the endcap calorimeter is at the large radius, large $\mathrm{z}$ corner. The photodetectors were placed at these points for several reasons: The next place where they could be located was after about 18 meters of cable length, resulting in substantial light loss. In addition, a design goal was the desire for the calorimeters to be completed and tested at the CMS surface assembly building before being lowered into the collision hall. The calorimeter design should therefore be self-contained and robust.

Because of the placement of the photodetectors, conventional photomultiplier tubes were unusable. Instead a recent development, hybrid photodiodes, HPD's, was adopted. The HPD is a proximity-focused device consisting of a vacuum envelop, a conventional photocathode, and a reverse-biased silicon diode. A high accelerating voltage (of order $10 \mathrm{kV}$ ) is supplied between the photocathode and the silicon diode. Photoelectrons emitted by the photocathode gain kinetic energy 
falling through the electric field. This kinetic energy is converted into electron-hole pairs when the photoelectron impacts the diode. The generated electric pulse is read off the diode, amplified, and sent to digitizing electronics. Typical gains for the HPD are from 1000 to 2000. Figure 7 shows the internal design of a HPD. Because the

\section{P25 7-Pixels}
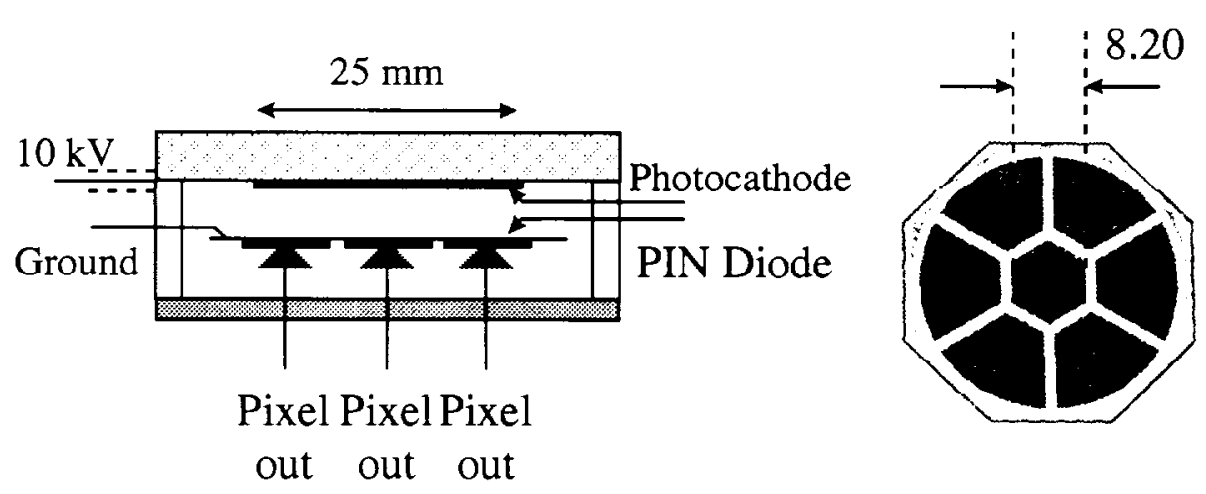

Figure 7: Internal design of a 7 pixel HPD.

low gain of the HPD requires the use of an associated amplifier, they are effectively noisier than conventional photomultiplier tubes. However HPD's are able to clearly detect minimum-ionizing particle transits of the calorimeter. Figure 8 shows a test beam measurement of the muon signal through 8 layers of scintillator using a HPD for readout 6 ).

Because of our reliance on radioactive sources for quality control and calibration, the HPD must be able to accurately measure DC currents (supplied by the radioactive sources). The HPD has a typical leakage current of about $1-5 \mathrm{nA}$, with a jitter in the current of only order $10 \mathrm{pA}$. The source-induced currents are of order $5 \mathrm{nA}$. With these conditions, the HPD's have been shown to make source current measurements of accuracy $1 \%$. 6 )

\section{Test Beam Studies}

There were a number of issues requiring exploration before the HCAL design could be optimized. Performance of hadron calorimeters operation in magnetic fields needed to be understood. Techniques for carrying calibrations from test beam conditions $(B=0)$ to operating conditions $(B=4 T)$ needed to be developed. Photodetectors which operate in high magnetic fields needed to be studied.

To understand these and other issues, a set of test beam measurements 


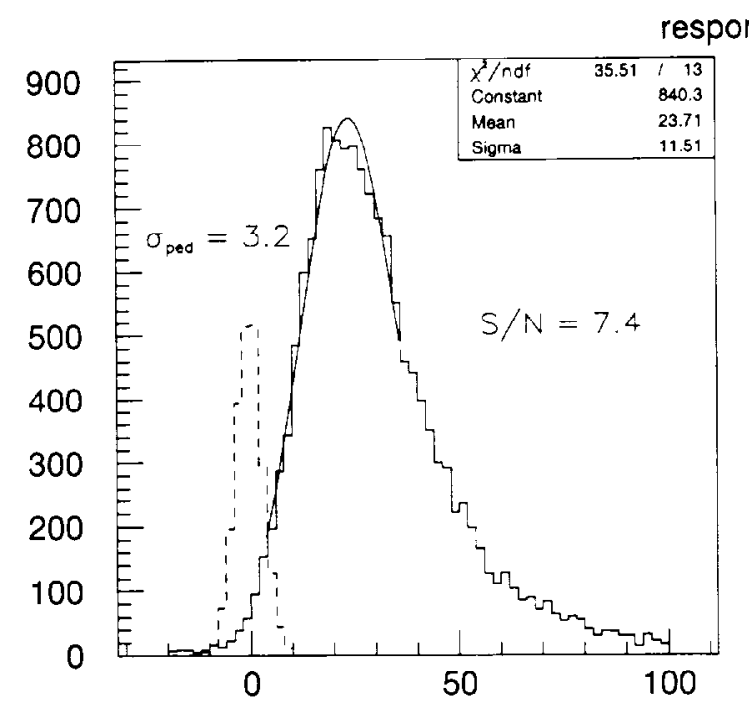

(a) HPD at $8 \mathrm{kV}$ (Stras Preamp)

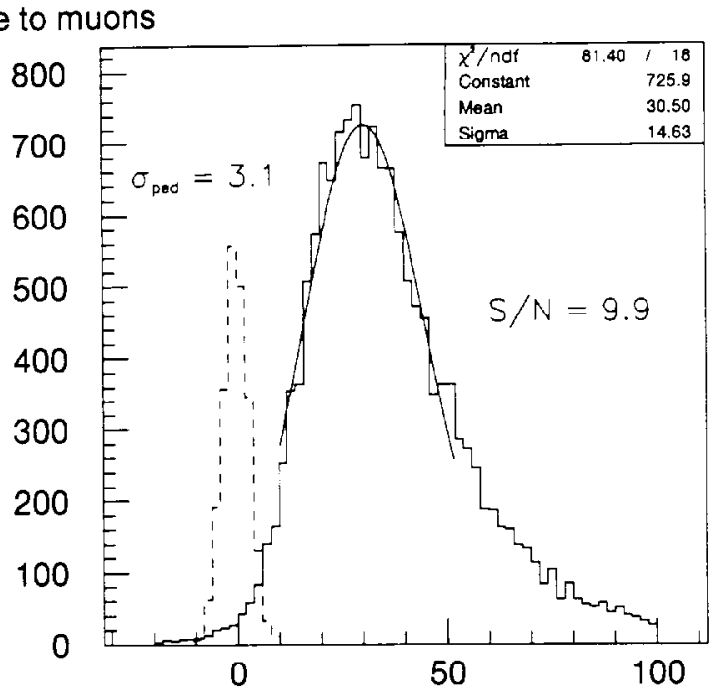

(b) HPD at $6 \mathrm{kV}$ (INR Preamp)

Figure 8: Test beam response for muons when viewed by an HPD. Light yield is approximately 10 photoelectrons. The dotted histograms are superimposed pedestal distributions.

were performed during 1995 and 1996. A "hanging file" calorimeter was constructed. This calorimeter could be easily reconfigured to study different absorber sampling and different scintillator arrangements. Copper absorber thickness of $2 \mathrm{~cm}$ or larger were available. The device had an active area of $64 \mathrm{X} 64$ centimeters, and could be arranged to be up to $10 \lambda$ deep. The transverse size was limited by the requirement to place it inside the EHS magnet in the CERN H2 beam line. This superconducting magnet has a cylindrical bore of 1.5 meters diameter and can supply magnetic fields of up to $3 \mathrm{~T}$. The test calorimeter could be exposed to magnetic fields either transverse or parallel to the beam.

Several sets of scintillators were built in 2 basic designs: One large tile of approximately $64 \times 64 \mathrm{~cm}$, read out by many WLS fibers; or $3 X 3$ arrays of $\sim 22 X 22$ $\mathrm{cm}$ tiles, each read by one WLS fiber. The light from each tile could be ganged together with the other tiles in the same layer to form individual longitudinal layers (to explore longitudinal shower development) or summed with other tiles in depth to form towers (to study transverse aspects and photodetector performance). The design of the test beam apparatus can be found in ref 7 ).

The 1995 exposure had the magnetic field parallel to the beam direction 
Pion, electron, and source response ratio vs $\mathrm{B}$ field

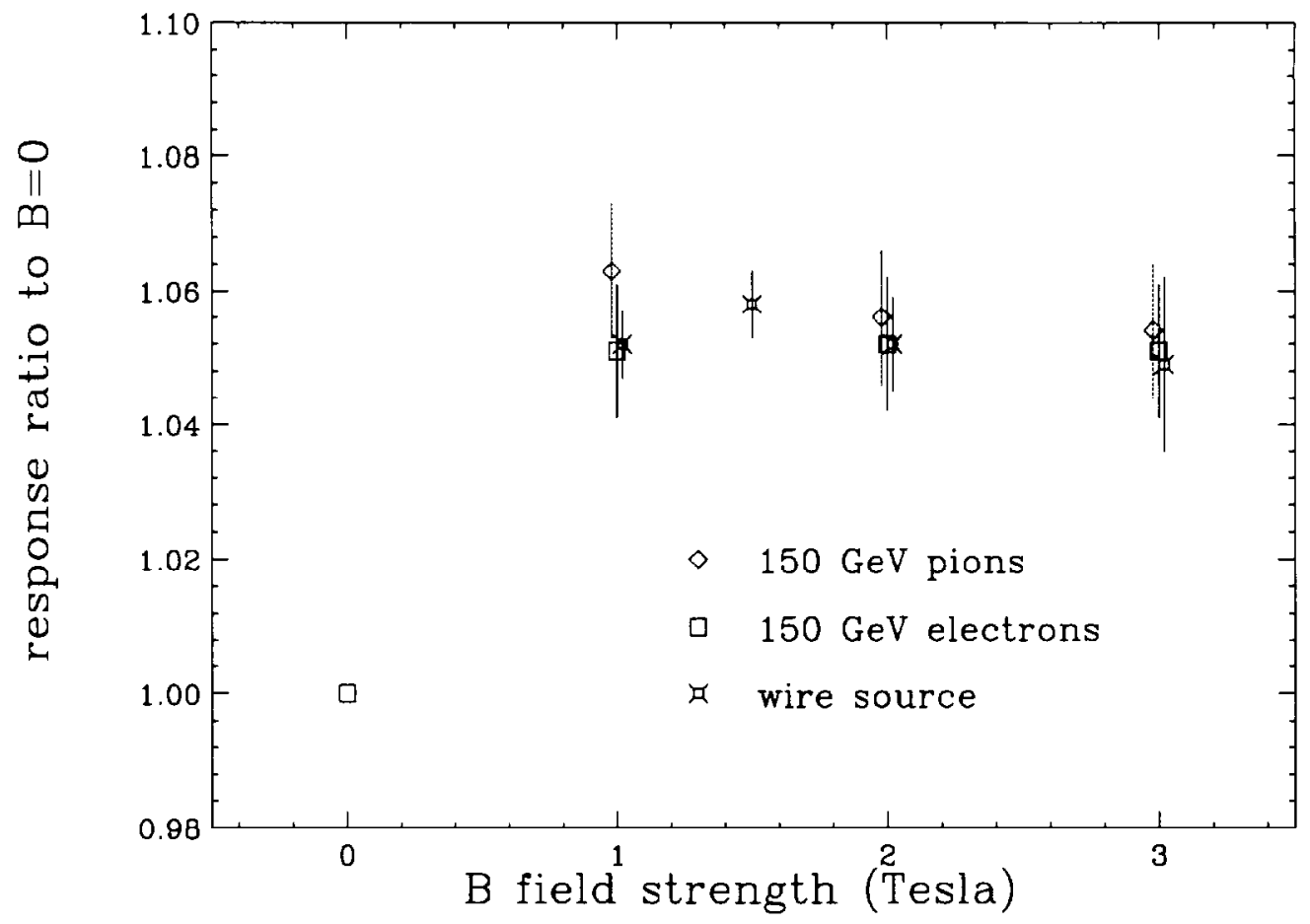

Figure 9: Normalized response for electrons and pions vs magnetic field. The field is parallel to the beam.

(the endcap configuration), while the 1996 orientation had the magnetic field perpendicular to the beam direction (the barrel configuration). Figure 9 shows results for the endcap configuration. Shown here are the normalized responses for pions and electrons as a function of magnetic field. There is about a $6 \%$ increase relative to $\mathrm{B}=0$. Also shown is the response of the moving wire source. We conclude that the observed change in response is simply the well known brightening of scintillator in a magnetic field. The response in the barrel configuration seems to be more complex. Earlier results 8$), 9)$ indicate that there are fundamental differences in pion and electron showers for transverse fields, and that $\mathrm{e} / \mathrm{pi}$ changes for $B \neq 0$. Monte Carlo results indicate that in the transverse configuration, electron showers in particular are sensitive to exact details of the transition between absorber and scintillator. Preliminary results from 1996 test beams agree with this conclusion. First results are arriving from analysis of the extensive sets of measurements taken in the summer of 1996. This analysis should be completed in the next several months. 


\section{$7 \quad$ Schedule}

The HCAL design will be finalized during fall 1996 and winter 1997. A full size pre-production prototype will be built during 1997, and tested at CERN test beams in 1998. In 1999 the production factory for HCAL will commence, with completion targeted for 2002 .

\section{References}

1. V. Gavrilov, et al, CMS Quartz Fiber Calorimeter, these proceedings.

2. Compact Muon Detector Technical Proposal, CERN/LHCC 94-38, p73

3. V.I. Kryshkin and A.I. Ronzhin, Nucl. Instrum. Methods, A247 (1986) 583

M.G. Albrow et al., Nucl. Instrum. Methods, A256 (1987) 23

4. G.W. Foster, J.Freeman and R.Hagstrom, Nucl. Phys. B, A23 (1991) 93

J. Freeman, et al., The CDF Upgrade Calorimeter, Proc. 2nd Int. Conf on Calorimetry in HEP, Capri, Italy, 1991

P. de Barbaro et al., Nucl. Instrum. Methods, A315 (1992) 317

P. de Barbaro and A. Bodek, A Compilation of Tile/Fiber R\&D Results, University of Rochester Preprint UR-1389 (1994)

5. J. Freeman and W. Wu, In situ Calibration of the CMS HCAL Detector, FNALTM-1984

6. P. Cushman, et al., Comparison of Hybrid Photodiodes and Avalanche Photodiodes as candidate transducers for CMS HCAL, CMS/TN 96-141, Proc. First Conference on New Developments in Photodetection, Beaune96, Beaune, France, June 24-28, 1996

7. H. Budd, CMS Central Hadron Calorimeter, Proc. 6th Topical Seminar on Experimental Apparatus in HEP, San Miniato al Todesco, Italy, May, 1996

8. J. Mainusch et al., Nucl. Instrum. Methods, A312 (1992) 451

9. V. Kryshkin, private communication. 